\title{
Developing role of medical audit advisory groups
}

\author{
Charlotte Humphrey, Diane Berrow
}

\begin{abstract}
Objectives-To investigate the approaches to audit of different medical audit advisory groups (MAAGs) and to consider the implications for evaluation of their activities and their developing role in the light of new priorities for clinical audit.

Design-Qualitative study based on semistructured interviews.

Setting-15 family health services authority (FHSA) districts in two English health regions.

Subjects-MAAG chairpersons and support staff and FHSA general managers and medical advisers in each district, totalling 68 subjects.

Main measures-Structures and activities of MAAGs; perceptions of the MAAG's role and its achievements compared with the initial brief in a health circular in 1990.
\end{abstract}

Results-The approaches of different MAAGs varied considerably: some concentrated on promoting audit and others were involved in a wider range of development activities. MAAGs assessed their progress in various different ways. The importance of collaborative working was recognised, but few interface audit projects had been undertaken. MAAGs had little contact with other quality assurance activities in the FHSA, and FHSA involvement in the MAAG strategy was variable, although MAAGs were taking steps to improve communication with the FHSA.

Conclusions-Major differences exist in the approaches taken by MAAGs and the roles they fulfil, which will make evaluation of their effectiveness a complex task. Already MAAGs are responding to changing expectations about audit and pressure for closer links with management.

(Quality in Health Care 1993;2:232-238)

\section{Introduction}

The 1989 white paper Working for Patients introduced medical audit as a central feature of the NHS reforms. ${ }^{1}$ In primary care, guidance was issued requiring each family health services authority (FHSA) to set up a medical audit advisory group (MAAG), whose function would be to direct, coordinate, and monitor medical audit activities in its area. ${ }^{2}$ MAAGs were to be medically led, most of their members being local general practitioner (GP) principals, but they would be accountable to the FHSA for carrying out their work (box).

\section{Accountabilities of MAAGs to FHSAs ${ }^{2}$ \\ - Instituting regular and systematic medical audit in which all practitioners take part \\ - Establishing procedures to ensure confidentiality for individual doctors and patients \\ - Establishing appropriate mechanisms to ensure that problems disclosed through audit are solved \\ - Providing a regular report on the general results of the audit programme}

Since 1989 thinking has changed about the nature and role of professional audit in the NHS. The Department of Health's recent policy statement on clinical audit ${ }^{3}$ exemplifies the new perspective. Medical audit is expected to give way to clinical audit, with audit becoming largely multiprofessional and spanning all aspects and sectors of care. Audit remains a professional activity, but there is increased emphasis on the influence of purchasers of health care, health service managers, and patients on the audit programme. In the light of the new priorities the department has emphasised the need to review the progress of the MAAG initiative along with that of the other audit programmes. As FHSAs move more into the role of purchasers and have to make difficult decisions about service priorities they are also increasingly concerned to know whether they are obtaining value for money from their MAAGs. ${ }^{4}$ Thus there is a desire both nationally and locally for an analysis of what the MAAGs have been doing.

MAAGs are new bodies with a new task. They have no precedent to work with, and no established historical relationship with, other agencies in primary care. In these circumstances it is hardly surprising that the variety of approaches adopted by different MAAGs has been noted as one of their most striking features. ${ }^{5}$ We report a qualitative study which set out to explore the nature and extent of this variation. The first aim was to find out what MAAGs were doing and how those people most directly involved perceived their tasks and achievements. By obtaining views of each study MAAG from various professional and managerial perspectives we sought to explore the degree of consensus about its role and to identify areas of disagreement. Further aims were to find out what mechanisms the MAAGs were using to measure their progress in promoting audit and to consider the implications of our findings for evaluating MAAGs themselves. 


\begin{tabular}{|l|}
\hline Variable characteristics \\
Geography/population \\
Large/small district \\
Rural/urban population \\
Affluent/deprived population \\
General practice characteristics \\
High/low proportions of singlehanded practices, \\
training practices, and GP fundholders \\
Presence/absence of a local academic \\
department of general practice \\
High/low profile local medical committee \\
Health service characteristics \\
Financially "losing"/"gaining" FHSA \\
Merged/separate FHSA/DHA \\
Overlapping/coterminous FHSA/DHA \\
boundaries \\
One/several local hospitals \\
\hline
\end{tabular}

\section{Subjects and methods}

Since our purpose was to explore variety rather than to establish frequencies we were more concerned to ensure coverage of a wide range of different approaches than to obtain a statistically representative sample. On these grounds we chose to study all $15 \mathrm{MAAGs}$ in two English regions instead of sampling randomly from the $90 \mathrm{MAAGs}$ in England as a whole. From preliminary discussions with staff in the two regions selected we were able to ascertain that the regions contained a range of districts with a wide variety of contrasting characteristics which seemed likely to influence the work of the MAAG (box).

Our initial intention was to seek interviews with the MAAG chairperson, MAAG support staff (clinical or lay), FHSA general manager, and FHSA medical adviser in each district. Names were obtained and responsibilities confirmed by telephoning the FHSA. In places where we learnt that the medical adviser had no contact with the MAAG, where

\begin{tabular}{|ll}
\hline $\begin{array}{l}\text { Interview topics and examples of subsidiary items of inquiry } \\
\text { Topic }\end{array}$ & $\begin{array}{l}\text { Example of subsidiary item } \\
\text { Extent of FHSA involvement in } \\
\text { deciding constitution }\end{array}$ \\
Membership of MAAG & $\begin{array}{l}\text { Roles and responsibilities of } \\
\text { different members }\end{array}$ \\
Group functioning & Communication within group \\
Financing & $\begin{array}{l}\text { Adequacy and conditions of } \\
\text { funding }\end{array}$ \\
Relationships with others & $\begin{array}{l}\text { Nature of FHSA-MAAG contact } \\
\text { Extent to which measurable } \\
\text { objectives set }\end{array}$ \\
Activities and methods used & $\begin{array}{l}\text { Most and least important activities } \\
\text { of the MAAG }\end{array}$ \\
Measurement of practice audit & Nature of data collected \\
activity & Perceived failures and \\
MAAG self evaluation & achievements \\
FHSA's perceptions of MAAG & Criteria for assessment \\
Future & Likely lifespan of the MAAG
\end{tabular}

structures varied, or where responsibilities had recently changed hands we followed local advice about whom to approach. We wrote to all individual subjects thus identified, asking them to agree to a confidential interview. When jobs were divided between a number of part time staff we asked to speak to one of the group or several together in the same interview.

We devised a semistructured interview schedule which we piloted with FHSA and MAAG staff in a district outside the study regions. The topics (box) were developed in consultation with an advisory group whose membership reflected the different interest groups included in the study. We used this interview schedule with all participants. When necessary during the interviews respondents were asked to clarify any differences between their personal views, those of others involved with the MAAG, and agreed MAAG strategies.

The interviews, which took one to two hours to complete, were recorded on audiotape and subsequently transcribed. Interview data were supplemented with additional information from all the MAAGs' annual reports and other relevant documents, where they were available. The data were analysed according to several themes selected on three different grounds: some were identified within the original brief for MAAGs; some have become relevant in the light of the more recent focus on clinical audit; and some emerged as important to understanding the work of the MAAGs during the interviews.

In this paper we draw on our findings to show how the MAAGs in our study had developed in different ways and how they viewed their progress in getting audit established. We consider how far they had taken on board changing views about the importance of multidisciplinary and interface audit, links with wider quality management, and management involvement in the audit agenda. Finally, we briefly discuss different criteria which might be used to evaluate the work of the MAAGs and consider the implications of our findings for any such assessments.

\section{Results}

Everyone approached agreed to be interviewed. In total we interviewed 68 people during the winter of 1992-3 (table 1). Two of the FHSA general managers and eight of the medical advisers interviewed were also MAAG members.

The titles, grades, and responsibilities of support staff varied considerably between MAAGs; here we refer to them generically as MAAG staff. In areas where views varied significantly between different categories of respondents we make this clear; where a reasonable consensus of opinion was found we have not distinguished between opinions. Respondents frequently referred to the MAAG as a single entity possessed of its own attitudes and perceptions; where it seems appropriate we have adopted this usage. 
Table 1 Subjects interviewed, by category, in 15 MAAGs in England

\begin{tabular}{|c|c|c|c|c|c|c|c|c|c|c|c|c|c|c|c|c|}
\hline & \multicolumn{16}{|c|}{$M A A G$} \\
\hline & $A$ & $B$ & $C$ & $D$ & $E$ & $F$ & $G$ & $H$ & $I$ & $\mathcal{F}$ & $K$ & $L$ & $M$ & $N$ & $O$ & \\
\hline $\begin{array}{l}\text { FHSA: } \\
\text { General manager } \\
\text { Chief executive } \\
\text { Medical adviser } \\
\text { Other directorate }\end{array}$ & $\begin{array}{l}+ \\
- \\
+ \\
-t\end{array}$ & $\begin{array}{c}+ \\
- \\
1 / 3^{\star} \\
-t\end{array}$ & $\begin{array}{l}- \\
+ \\
2+ \\
-t\end{array}$ & $\begin{array}{l}- \\
+ \\
+ \\
-t\end{array}$ & $\begin{array}{c}2+ \\
- \\
+ \\
-t\end{array}$ & $\begin{array}{l}+ \\
+ \\
-\dagger \\
+\end{array}$ & $\begin{array}{l}+ \\
- \\
+ \\
-t\end{array}$ & $\begin{array}{l}+ \\
- \\
+ \\
-t\end{array}$ & $\begin{array}{l}+ \\
+ \\
+ \\
+\end{array}$ & $\begin{array}{l}+ \\
- \\
+ \\
-t\end{array}$ & $\begin{array}{l}+ \\
- \\
- \\
+\end{array}$ & $\begin{array}{l}+ \\
+ \\
+ \\
+\end{array}$ & $\begin{array}{l}+ \\
- \\
+ \\
-t\end{array}$ & $\begin{array}{c}+ \\
\overline{2} \\
22^{\star} \\
-t\end{array}$ & $\begin{array}{l}+ \\
- \\
-t \\
-t\end{array}$ & $\begin{array}{r}14 \\
4 \\
13 \\
3\end{array}$ \\
\hline $\begin{array}{l}\text { MAAG: } \\
\text { Chairperson } \\
\text { GP facilitator } \\
\text { Lay support staff }\end{array}$ & $\begin{array}{l}+ \\
- \\
+\end{array}$ & $\begin{array}{l}+ \\
+ \\
+\end{array}$ & $\begin{array}{l}+ \\
- \\
+\end{array}$ & $\begin{array}{c}+ \\
1 / 7^{\star} \\
-\end{array}$ & $\begin{array}{l}+ \\
+ \\
-\end{array}$ & $\begin{array}{l}+ \\
+ \\
+\end{array}$ & $\begin{array}{l}+ \\
- \\
+\end{array}$ & $\begin{array}{l}+ \\
+ \\
+\end{array}$ & $\begin{array}{l}+ \\
+ \\
+\end{array}$ & $\begin{array}{l}+ \\
+ \\
-\end{array}$ & $\begin{array}{c}+ \\
4_{4}^{\star}\end{array}$ & $\begin{array}{c}+ \\
2 / 2^{\star}\end{array}$ & $\begin{array}{l}+ \\
- \\
+\end{array}$ & $\stackrel{+}{1 / 4^{\star}}$ & $\begin{array}{l}+ \\
+ \\
+\end{array}$ & $\begin{array}{r}15 \\
4 \\
15\end{array}$ \\
\hline Total & & & & & & & & & & & & & & & & 68 \\
\hline
\end{tabular}

HOW MAAGS HAVE DEVELOPED

The MAAG circular was primarily concerned with the organisational structure within which audit should be undertaken, rather than the nature of the audit process. ${ }^{2}$ The original guidelines deliberately left room for local interpretation, on the assumption that approaches would differ from place to place and evolve as experience was gained.

The MAAGs in our study had conformed fairly closely to the original recommendations, as far as they went. All were numerically dominated by GPs (table 2); however, 12 of the 15 had extended their membership to include at least one FHSA representative. They were all heavily involved in providing training, support, and facilitation for their constituent practices, with a variety of different approaches; all had some links with the wider medical education system, all had developed effective methods of protecting confidentiality, and all reported regularly on their activities to the FHSA. But within these commonalities there were important differences between styles and activities reflecting the substantial room for manoeuvre within the original guidelines and the impact of widely differing local circumstances on the nature of the task faced by the MAAGs and the shape of the local response. For example, the MAAGs varied in how they perceived their role in relation to management, some seeking to provide a "buffer" between local general practice and the FHSA, others serving as a "bridge"; how they defined their function, some choosing a narrowly defined focus on

Table 2 Comparison of guidelines for membership and findings for 15 study MAAGs

Guidelines Study MAAGs

Precise size to be determined locally. Normally "No more than 12 members who are medically qualified"

Majority of members to be local GP principals One hospital consultant

One public health doctor

Other health professionals might be co-opted

No mention of lay representation

No mention of FHSA representation

MAAG might employ GP facilitators

No mention of lay support staff

Range 7-15 medically qualified members

Range 6-12 GPs

$\geqslant 1$ Consultant (14 MAAGs)

$\geqslant 1$ Public health doctor (13 MAAGs)

Nurse member (1 MAAG)

Lay member (1 MAAG)

FHSA general manager ${ }^{\star}(2 \mathrm{MAAGs})$

FHSA medical advisers (8 MAAGs)

Other FHSA representation (2 MAAGs)

GP facilitators (4 MAAGs)

Lay support staff (11 MAAGs) audit, others assisting practices to meet a wide range of developmental needs; and how proactive they were in setting the local agenda for audit. The FHSA managers likewise differed in their perceptions of the MAAG's role, some regarding it as confined to providing audit support to general practice, others as a potential source of professional advice to the health authority across a wide range of service issues. They varied in how much importance they attached to the MAAG and what they committed to it in office support or additional funds beyond the basic budget allocation. Almost half the MAAGs had access to funding from other FHSA budget areas to help support audit. As a result of such differences the problems facing the MAAGs and their criteria for measuring success were also very variable among districts.

From our findings we constructed three models of MAAGs (box), whose characteristics were chosen, not for their particular dominance - no dominant combinations could be identified - but to illustrate the complexity of the variation. Our sample contained 15 MAAGs, each of which was unique in important ways; presumably in England as a whole there are 90 different versions. The extent of these differences limits the observations that can be made about the MAAGs as a homogeneous group.

\section{ESTABLISHING AUDIT}

The first and main task for which the MAAGs were accountable in the circular was "the institution of regular and systematic medical audit in which all practitioners take part."2

All the MAAGs in our study had made efforts to evaluate their progress in audit and were becoming more systematic and advanced in their methods of categorising practice audit activity. Many had developed their own systems of assessment, others were using adapted versions of a model developed by the Oxford MAAG. ${ }^{6}$ However, many MAAGs still had incomplete information about the audit activity in their practices, especially those that were unwilling to press their practices for details, those whose strategies did not entail regular or comprehensive practice visits, and those that had adopted a decentralised model of patch based working. MAAG staff also commented on the difficulty of keeping their information up to date and ensuring that practice visitors filled out assessment forms consistently. Therefore, most staff had doubts about the accuracy and validity of their own data. These problems apart, no consistency existed among the MAAGs in the information recorded about practice activity. It would not be possible, therefore, to produce an accurate aggregate measure of the degree of progress towards the objectives.

Nevertheless, all the people we interviewed from MAAGs and most FHSA respondents felt that progress was being made: there was general confidence that more practices were doing audit; the range of practice team members involved had widened; the topics 


\section{Contrasting models of MAAGs}

$M A A G A$

Setting: Large suburban district with a minority inner city population. Well established teaching practices in affluent areas and struggling singlehanded practices. Financially "losing" FHSA.

Membership: Based on shadow audit group set up by local medical committee. Commitment to including "normal" GPs. No FHSA presence on MAAG.

Siting and support: Administrative assistant based in MAAG member's practice.

Finance: $\quad £ 80000$ basic budget allocation from FHSA.

Philosophy: Non-directive, led by local GPs' interests. Willing to advise practices on wide range of professional issues. Not keen to provide practical help in areas of FHSA responsibility.

Strategy: Highly devolved patch based support led by GP facilitators.

Perceived success: Developing the trust and interest of previously sceptical local general practice.

Pressing issues for $M A A G$ : Responding to diverse needs of local practices; maintaining independence from FHSA.

Pressing issues for FHSA: Improved communication with MAAG over its strategy; seeking evidence of value for money.

\section{$M A A G B$}

Setting: Medium sized, city based district. Strong academic department of primary care. Single central hospital. Coterminous FHSA/DHA boundaries.

Membership: Set up by general manager in consultation with academic GPs. High profile academic leadership with commitment to excellence. General manager is observer at MAAG meetings.

Siting and support: Research coordinator based in academic department and several audit assistants.

Finance: $£ 60000$ basic budget allocation from FHSA; $£ 45000$ over two years from externally funded projects.

Philosophy: Strong commitment to educational leadership. Keen to help practices with audit but not wider development issues.

Strategy: Organises districtwide projects on topics selected for their value in teaching about audit. Lay support staff provide practices with technical help and advice in writing proposals for funding.

Perceived success: Obtaining substantial external funding for projects, several publications, and a national reputation for rigorous audit.

Pressing issues for MAAG: Maintaining project funding and coordinating MAAG funding from multiple sources.

Pressing issues for FHSA: Keeping MAAG down to earth and focused on practical local issues rather than research projects.

\section{$M A A G C$}

Setting: Small urban/rural district. FHSA/district health authority recently merged.

Membership: Set up by FHSA medical adviser with public health background. Members chosen for enthusiasm and technical expertise. Medical adviser sits on MAAG.

Siting and support: Audit facilitator based in FHSA.

Finance: $£ 45000$ budget allocation from FHSA. Free accommodation and office support. Access to GMS and FHSA "slippage" monies for practice support on an ad hoc basis.

Philosophy: Pragmatic commitment to improving services by whatever means available.

Strategy: Mixture of MAAG initiated audit projects addressing local priorities; computer based group audits and facilitation of local practice development (help with age-sex registers, teamworking, etc).

Perceived success: Working with FHSA to develop practice information systems and compatibility of computer systems for audit.

Pressing issues for $M A A G$ : Fear of neglect of audit by the new joint health authority with its wider agenda.

Pressing issues for health authority: Developing multidisciplinary

collaboration over a wide range of quality issues; identifying opportunities for transfer of resources to primary care. audited were becoming more appropriate; audit skills had improved; and interest in audit had increased and fear had diminished. Some MAAGs could document these changes in great detail with evidence from their records; in others the assessment depended on a wide variety of indicators such as comments from practices, attendance at meetings, requests for MAAG help, etc. Several of the MAAG staff we interviewed, however, questioned the extent to which the MAAG could take the credit for these developments. Some felt that, in part at least, they were observing and documenting changes that would have happened anyway.

At the same time the MAAG respondents clearly appreciated where the MAAG's limits lay. All knew of practices that were not auditing and seemed unlikely to start. Some acknowledged that they had given up on a minority of the most resistant practices (often with the tacit agreement of the FHSA), believing their efforts were better placed where they were more likely to be successful. Among the practices that were doing audit, all the MAAGs were aware of instances in which one keen partner or a member of the practice staff was carrying the audit brief for the practice as a whole. The direct involvement of all practitioners, as opposed to practices, was seen by most respondents as a distant or unrealistic objective.

In the winter of 1992-3 most of the MAAGs in the study were still fully engaged in teaching about audit and getting practices started. Encouraging practices to move beyond data collection to complete the audit cycle was recognised to be the next major task and in many ways the acid test of the MAAG's worth. One MAAG chairperson spoke for many when he acknowledged that much of the current activity was not useful as it stood. "If it stays like this," he said, "we may well look back in a few years' time and say the whole thing was a failure."

The MAAG respondents also recognised the difficulties of assessing and demonstrating effective change, even supposing it could be achieved. One problem was that they did not necessarily know where changes had occurred since their commitment to confidentiality precluded access to audit results unless these were volunteered by the practice. A further problem was that, even where beneficial change was known to have taken place, it could not necessarily be acknowledged publicly without compromising the privacy of the practice. These constraints aside, beneficial effects on patient health are notoriously difficult to identify in primary care. Most MAAGs therefore relied on interim indicators of effectiveness such as changes in practice behaviour. On this basis, and using their informal knowledge of the practices, most MAAG staff we spoke to were able to produce a list of examples of beneficial change. However, there was an awareness that the changes were not always achieved in "the right way" - that is, through completing the audit cycle. 
INTERFACE AUDIT AND MULTIDISCIPLINARY WORKING

The original circular required MAAGs to establish links with public health medicine and consultants associated with hospital medical audit with a view to auditing services bridging hospital and community health services and primary care. Within primary care co-option of other team members was suggested. Nevertheless, medical audit among GPs was clearly predicted to be the major focus of activity.

The study MAAGs had their prescribed complement of hospital and public health consultants, but these members were of varying importance in the group. A few members were strongly engaged with the MAAG, others had only peripheral involvement and rarely turned up to meetings. Relatively few interface audit projects had been undertaken. Those that flourished were usually large scale projects that had obtained additional separate funding. The initiative for such projects tended to come from either one committed individual member of the MAAG or a particular confluence of circumstances, such as local interest and skill in a particular subject and opportunistic links between MAAG members and hospital staff. Such projects had clearly been easier to set up in districts with fewer hospitals and simpler local referral patterns.

MAAG respondents were well aware that success in implementing audit depended on the involvement of the whole professional team. Practice staff were encouraged to participate in discussions about audit at practice visits and in educational activities organised by the MAAG. However, commitment to multidisciplinary working had not extended to having a multidisciplinary MAAG. Many of the MAAGs had discussed co-opting other primary care staff as members of the group, but with one exception they still remained entirely medical in their professional membership.

The MAAG respondents did view interface audit and collaborative working as important, but generally they saw these as goals to pursue once audit was going well among GPs. Many regarded their present GP centred approach as the obvious first step in a development model which starts with the core professional group, progresses to include the practice team, and subsequently expands to encompass the wider primary health care team and community and hospital services. On the other hand, a minority of MAAGs were already taking a more eclectic approach, seeking to tap into enthusiasm for audit wherever it was to be found. These MAAGs had learnt from experience that there was often more commitment to audit among team members other than the GPs. They were also finding that GPs themselves were interested in carrying out audit at the interface with secondary care. In part this reflected GPs' concerns about the services their patients were receiving elsewhere; many were also keen to develop new skills and extend the care they provided - for example, in shared care for chronic diseases.

WIDER QUALITY MANAGEMENT

Initially, medical audit was seen as clearly separate from wider issues of quality, and it was anticipated that the FHSAs would develop independent mechanisms to consider quality. Since then they have become involved with various quality initiatives including the patients' charter, total quality management projects, and British Standard 5750.

Most MAAGs in our study had no links with other quality initiatives in the FHSAs and did not forsee any. In a few cases, where the MAAG office was based in the FHSA, informal contact between MAAG staff and members of the quality assurance directorate had led to joint working on specific projects. Some of the MAAG staff involved in such collaborations, however, felt uneasy about the propriety of this association.

Formal quality assurance initiatives apart, many MAAGs had expanded their own brief to encompass several wider quality issues. For example, they were using their growing experience and knowledge about local resources to provide a significant amount of informal help to individual practices with various personal, clinical, or organisational problems often only indirectly to do with audit. In this respect most of the MAAG respondents acknowledged a support function far wider than their official role in promoting audit. Some felt this was an undesirable expansion which distracted energy and attention from the MAAG's proper purpose and led to a dangerous blurring of responsibilities between MAAG and FHSA. In some districts FHSA staff shared this view and accepted the continuing need for a limited, professionally led focus exclusively on audit and were successfully using other routes to involve professionals in their service development activities. In others MAAG respondents were interested in moving cautiously towards a role as a professional arm of the FHSA, offering advice on a wide range of practice and service development issues. Several of the FHSA managers and medical advisers were keen to suggest possible areas of collaboration with the MAAG (box). There

Suggested service development roles
for MAAGs
Providing advice to FHSAs in:
- Evaluating changes in service provision
- Identifying opportunities for service
innovation
- Identifying needs
- Evaluating demands
- Developing acceptable systems of assessment
- Developing standards
- Investigating local problems
- Promoting local strategies
- Developing other quality initiatives


was no consistent relation within districts between the views of the MAAG and FHSA on the role of the MAAG.

\section{MANAGEMENT INVOLVEMENT}

The original brief proposed joint discussions between MAAG chairpersons and FHSA general managers to agree the programme and scale of medical audit activity, ${ }^{2}$ though by implication this was more concerned with setting the budget than agreeing the content of the MAAG's work. There was no mention of FHSA representation on the MAAG, although the FHSA had the option of suggesting members.

In 13 of the 15 study MAAGs, FHSA staff regularly attended meetings, some only as observers, but most with full membership status. Despite this presence management involvement in the MAAG's strategy was very variable. Several of the medical advisers were involved with the MAAG in a personal capacity rather than as representatives of the FHSA, and others chose to stand back from the decision making and take a more advisory role. Some general managers had played a major part in establishing the MAAG and subsequently stepped back; others had had relatively little involvement and were still seeking to establish dialogue.

There was a strong sense of growing interest among the FHSAs in negotiating with their MAAGs to ensure that national and local priorities were taken into account in planning work. The MAAGs were aware of this pressure and many had already taken steps to improve communication with the FHSA to identify common interests and increase their understanding of each other's needs. Although not prepared to be told what to do by the FHSA, they accepted the need to justify their funding not only in terms of effectiveness but also relevance to the authority's concerns. In many cases the interests of the FHSAs, MAAGs, and their constituent practices had emerged as quite compatible, simply because all were preoccupied with the same current issues, such as the health promotion banding system and the Health of the Nation priorities. All the MAAGs were committed to respecting the right of practices and individuals to choose their own audit topics, should they wish. A few MAAGs were unwilling to offer any directive leadership at all, and these were the districts that seemed to be having the greatest difficulty in reconciling the views of MAAG and FHSA.

\section{Discussion}

We have explored the considerable variety between the approaches of different MAAGs and also identified some features they share and discussed some of the common directions they seem to be developing. Finally we briefly consider some of the implications of our findings for assessment of the value of MAAGs.

Nationally, concern might be expected to concentrate on the MAAGs' demonstrated effectiveness in promoting audit, inasmuch as this was the purpose for which they were created. However, the range of additional functions that some of the MAAGs had successfully taken on means that an evaluation on the basis of the audit work alone would be incomplete. An important strength of the way the MAAG guidelines were formulated was the opportunity for local innovation and the resulting exploration of previously unconsidered ways of working for example, in providing professional advice on service issues. Such local developments might be evaluated on their own account and their adaptability for use elsewhere considered.

An evaluation of the MAAG initiative would have to weigh the cumulative achievements and shortcomings of all the different models represented. Earlier we commented on the problems of aggregating evidence of the progress of MAAGs and interpreting their achievements in promoting audit. Insofar as each of the MAAGs in our study was working with different priorities in different circumstances it would be equally difficult, and arguably inappropriate, to compare their approaches with a view to saying which works best. This was certainly the view of our respondents. Although many were confident of the advantages of their own approach over those of other districts that they knew about, they all accepted that no single way of working would be applicable everywhere.

In contrast, local evaluation of any individual MAAG will inevitably be influenced by the impact and perceived appropriateness of the particular approach that it has taken. Although acknowledging the audit brief of the MAAGs, some of the FHSA managers in our study were equally (sometimes even more) interested in evidence of the MAAG's ability to help them deal with other pressing issues on their own agendas. In those study districts where the views of the FHSA and MAAG of the MAAG's role differed, the perceptions of its value tended to be equally at odds.

In conclusion, when the MAAGs were set up it was not known whether their structure was appropriate to the task or how they would work. Since then those involved with MAAGs have developed a wealth of skills and understanding about what is possible and how it can be done. The MAAGs in our study had clearly provided a focus for sustained thinking about the value and limitations of audit and its links with wider service development activities. Without this focus it seemed unlikely that local understanding and discussion of these issues would have progressed so far as it had.

The past three years have seen great changes in primary health care services and in priorities for audit. Consequently, demands on the MAAGs have also changed. The new objectives of collaboration between different services and closer links between professions and management are arguably more difficult to meet and at least as controversial as the original medical audit brief. Nevertheless, the MAAGs in our study had already begun to respond to these changing expectations and 
were making progress on both fronts. Despite this evidence of flexibility most people interviewed recognised that further development might entail more fundamental modifications to the MAAGs. Although they were concerned not to leave the original business of the MAAGs unfinished, many of them were already thinking about new names and structures for taking audit forward.

We thank the MAAG and FHSA staff who participated. The study was supported by a grant from the Department of Health.
1 Secretaries of State for Health, Wales, Northern Ireland, and Scotland. Working for patients. London: HMSO, 1989. (Cm 555.)

2 Department of Health. Health service developments - working for patients. Medical audit in the family practitioner services. London: HMSO, 1990. (HC(FP) (90)8.)

3 Department of Health. Clinical audit: meeting and improving standards in health care. London: NHS Management Executive, 1993.

4 Wall C. Medical audit - value for money. Audit Trends 1993;1(2):43-5.

5 Spencer J. Audit in general practice: where do we go from here? Quality in Health Care 1993;2:183-8.

6 Derry J, Lawrence $M$, Griew K, Anderson J, Humphreys J, Pandher KS. Auditing audits: the method of Oxfordshire Medical Audit Advisory Group. BMF 1991;303:1247-9. 